\title{
Les tuputupua: ancêtres protecteurs et animaux tutélaires aux Tuamotu
}

The tuputupua: protective ancestors and animal guardianship in Tuamotu

\section{Florian Prévost}

\section{(2) OpenEdition}

12 Journals

\section{Édition électronique}

URL : http://journals.openedition.org/jso/7894

DOI : $10.4000 /$ jso.7894

ISSN : $1760-7256$

Éditeur

Société des océanistes

\section{Édition imprimée}

Date de publication : 15 décembre 2017

Pagination : 329-344

ISSN : 0300-953x

\section{Référence électronique}

Florian Prévost, « Les tuputupua : ancêtres protecteurs et animaux tutélaires aux Tuamotu », Journal de la Société des Océanistes [En ligne], 144-145 | 2017, mis en ligne le 15 décembre 2019, consulté le 15 mars 2021. URL : http://journals.openedition.org/jso/7894 ; DOI : https://doi.org/10.4000/jso.7894

\section{(c) (i) $\odot$}

Journal de la société des océanistes est mis à disposition selon les termes de la Licence Creative Commons Attribution - Pas d'Utilisation Commerciale - Pas de Modification 4.0 International. 


\section{Les tuритириа: ancêtres protecteurs et animaux tutélaires aux Tuamotu}

par

Florian PREVOST ${ }^{*}$

\section{RÉSUMÉ}

Aux Tuamotu, Eugène Caillot fut l'un des premiers à fournir des informations sur les pratiques liées au culte des ancêtres. Si les études sur le sujet ont permis de mettre en lumière certains aspects de la conception de la vie après la mort des Paumotu, d'autres éléments sont restés plus confidentiels. Ainsi, la réincarnation des ancêtres sous une forme animale, bien que mentionnée, n'a jusqu'ici pas fait l'objet d'une étude à part entière. Pourtant dans les mémoires des autochtones, les récits relatifs à ces entités qu'ils ont nommées tuputupua, sont encore vivaces. Ce travail propose donc d'étudier les interactions entre tuputupua et le groupe de descendance qui leur est affilié. Pour se faire, nous nous sommes essentiellement basé sur un corpus de traditions orales de l'aire dialectale Tapuhoe, correspondant au centre de l'archipel des Tuamotu.

MotS-CLÉs : Tuamotu, tradition orale, ancestralité, animaux, totémisme

\begin{abstract}
In the Tuamotu archipelago, Eugène Caillot was one of the first to give information about practices associated with the ancestors' cult. If studies on the matter were able to enlighten certain aspects of Paumotu's life after death conception, some elements remained more confidential. Indeed, reincarnation of ancestors in an animal shape, though mentioned, had not constituted a specific study to this day. However tales about those entities, Paumotu had named tuputupua are still vivid in their memories. This work proposes to study interactions between tuputupua and affiliated group of descents. In order to do so, we focused on a collection of oral traditions issued from the linguistic area of Tapuhoe which is located at the center of the Tuamotu archipelago.
\end{abstract}

Kerwords: Tuamotu, oral tradition, totemism, animals, ancestrality

Comme dans toutes sociétés traditionnelles, les habitants des Tuamotu étaient, avant le contact avec le monde occidental, en lien étroit avec leur environnement naturel. Ce lien s'exprimait d'un point de vue matériel, par l'exploitation de ses ressources terrestres et marines, mais également sur un plan spirituel. Une croyance que nous allons étudier confere, en effet, dans la zone qui nous intéresse, un lien plus étroit encore entre l'homme et certaines espèces animales qui l'entourent. Ce type de croyance fait irrémédiable-

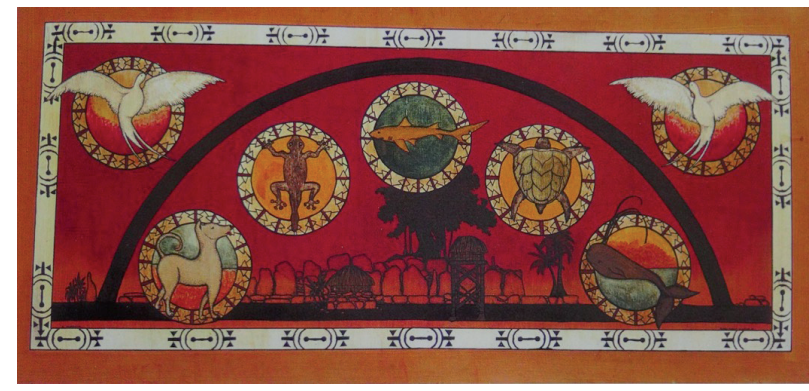

Figure 1. - «E'aumakua», tableau de Bobby Holcomb tiré de l'ouvrage Visions polynésiennes (1992) 


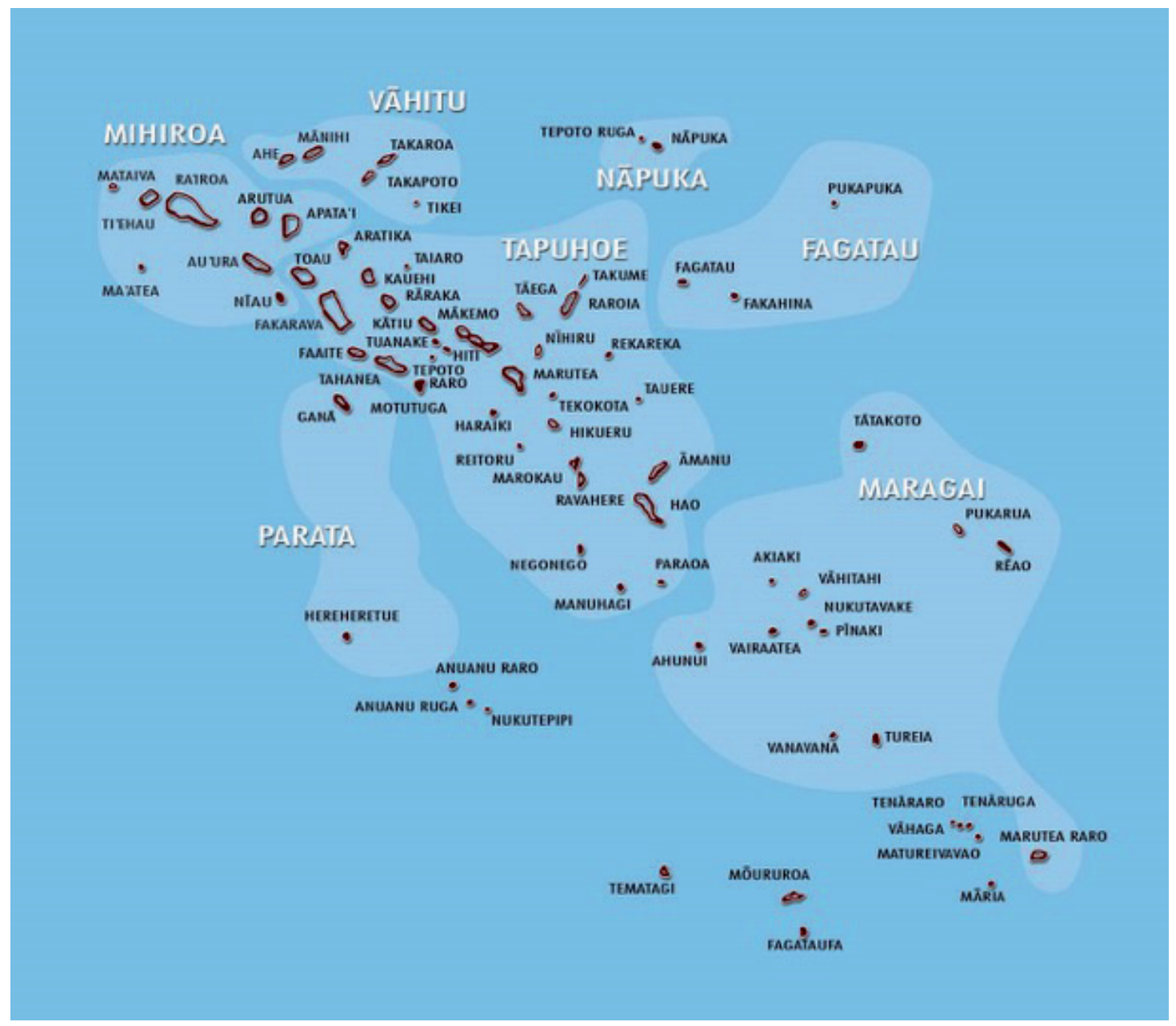

Figure 2. - Carte des Tuamotu et localisation des sept aires dialectales (Association Te reo o te Tuamotu, d'après Stimson, 2008)

ment penser au totémisme ; toutefois, nous éviterons autant que faire se peut de calquer une grille d'analyse préconçue sur les récits que nous étudierons. Jean Guiart rappelait en effet que le totémisme a longtemps été une

" catégorie socio-religieuse [...] plaquée artificiellement sur le monde entier. » (Guiart, 2012 : 27)

Le même auteur ajoutait ainsi que :

"la référence permanente aux totems indiens évitait de se préoccuper de savoir si un tel vocabulaire était ou non adapté à l'analyse de toute utilisation de symboles animaux ou végétaux. » (Guiart, 2012 : 26)

L'intérêt de cette croyance dans le contexte des Tuamotu est qu'elle a su traverser les époques, sans pour autant rester figée sous sa forme initiale. Notre travail évoquera ainsi tout autant les temps pré-européens que l'époque actuelle. En tentant de la définir, nous dirions qu'il s'agit de la transformation d'un ancêtre en un animal après la mort ou plutôt de la pénétration de l' " esprit » ${ }^{1} \mathrm{du}$ mort dans un corps animal. Ce dernier est chargé dès lors de veiller sur et de protéger le groupe de parenté auquel il est rattaché et avec qui il entretient une communication pouvant prendre des formes diverses. C'est cette créature animale, qu'elle soit perçue comme habitée par l'« esprit » du défunt ou comme la réincarnation d'un ancêtre, que nous nommerons ci-après tuритириа.

Après quelques éléments de linguistique et un rappel des écrits existants sur le sujet aux Tuamotu, nous analyserons successivement trois traditions orales s'y rapportant afin d'en dégager les principales caractéristiques et les originalités. Ces traditions sont toutes issues de l'aire dialectale Tapuhoe, située au centre de l'archipel des Tuamotu ${ }^{2}$. Nous chercherons par la suite à

1. Nous mettons entre guillemets car nous verrons que le terme d'esprit n'est pas tout à fait pertinent dans le contexte océanien.

2. Elles ont été récoltées dans le cadre d'un travail de thèse en anthropologie en cours, l'auteur résidant dans la zone depuis 2009. 
mettre ces récits en lien avec ceux similaires des autres sociétés du Pacifique dans le but d'appréhender leur degré d'imbrication.

\section{Premiers éléments d'analyse}

\section{Définition et étymologie du terme tuputupua}

Un tuputupua est défini par Frank Stimson comme un

"être surnaturel de l'au-delà, une sorte d'esprit-dé-
mon ayant un pouvoir individuel inspirant la peur et
l'effroi. » (Stimson et Marshall, 2008 : 575)

Il rapproche ainsi le tuputupua du terme européen "démon ", l'être décrit se caractérisant essentiellement par son pouvoir de nuisance. Cette définition lui a été suggérée par les informations recueillies auprès de Paea-a-Avehe ${ }^{3}$ de Anaa, pour qui les tиритириа " effectuent seulement des actions néfastes. Ils se saisissent des esprits des morts et les emportent afin qu'ils soient mangés" (Stimson, 1933: 26). D’une manière générale, les informateurs de Stimson s'accordent pour décrire les tиритириа comme des entités malfaisantes ${ }^{4}$ (vaerua kirokiro) évoluant dans le $P \bar{o}$, le monde de l'invisible et des morts. Certains comme les Paki-raho-nui étaient très attirants et cherchaient à dévier de leur route l'esprit des morts afin qu'ils n'atteignent pas leur dernière demeure, d'autres apparaissaient en rêve pour terrifier certaines personnes durant leur sommeil (Stimson, 1933: 26).

Malgré la relative uniformité de ces descriptions, Stimson semble hésitant sur la définition de l'entité tиритириа, lui donnant un nombre élevé de synonymes potentiels ${ }^{5}$. Aujourd'hui, le sens du terme tel qu'il est défini par ses informateurs ne semble plus d'actualité. C'est pourquoi dans notre propos, nous nous focaliserons davantage sur l'acceptation moderne du terme (vue en introduction), tout en analysant plus loin dans quelle mesure le sens de celui-ci a pu évoluer dans le temps.

L'anthropologue Frédéric Torrente, étudiant les Tuamotu, définit quant à lui les tuputupua comme des " êtres protecteurs des humains, des entités relevant du règne animal ", mais pouvant également être végétales ou minérales (Torrente, 2012 : 222). Éric Conte, dans sa thèse consacrée à l'atoll de Napuka, indique que :
" [ce] terme peut à la fois désigner des esprits circulant sans être incarnés et des animaux terrestres ou marins que l'on considère comme habités par l'esprit d'un ancêtre. » (Conte, 1988 : 162)

\section{Il ajoute que :}

« certains sont des protecteurs, d'autres plutôt désignés sous le nom de vaka, sont des moyens de transports ou des messagers. »(Conte, 1988 : 162)

Aujourd'hui, notons que certains habitants des Tuamotu avec qui nous nous sommes entretenu seraient tentés de traduire tиритириа par " ancêtre éloigné ». Pour eux, il serait donc synonyme de tupuna, terme désignant habituellement les ancêtres, tant dans la zone que dans l'ensemble polynésien, mais une plus grande ancienneté leur conférerait une dimension sacrée que n'auraient pas les tupuna.

Cette variété de sens pour un même terme peut s'expliquer par l'existence au sein de l'archipel des Tuamotu de plusieurs aires dialectales. Ainsi dans L'Atlas linguistique de Polynésie française, les auteurs notent que si le terme tuputupua est connu dans plusieurs zones de l'archipel, son sens differe quelque peu. Dans le sud de l'archipel, ainsi qu'à Tahiti, il serait synonyme de revenant et du terme tahitien 'aiaru (" revenant qui vient infliger une maladie à quelqu'un de la famille ", Fare Vana’a) (Charpentier et François, 2015 : 1090-1091). Dans le centre des Tuamotu, ainsi qu'à Rapa, il désignerait plutôt un fantôme et serait ainsi synonyme de tupapa'u, terme générique connu dans la plupart des archipels de Polynésie française.

En terme d'étymologie, le mot pourrait se décomposer comme suit tири / tириа. Тири indique le fait d'apparaître, de venir à la vie, d'émerger tandis que tириа désigne une personne exaltée "comme un roi, ou un héros renommé " ou encore "une sorte de démon" (Stimson et Marshall, 2008 : 575). Une traduction pourrait donc être : celui qui apparait sous la forme d'un démon ou d'une personne exaltée, ce qui est en accord avec notre sujet. Firth écrit la même chose à Tikopia où, pour lui, le terme "tupu" "indique le processus de transformation d'un être spirituel en sa manifestation matérielle" (Firth, 1930 : 304). C'est ce terme qui est utilisé dans un texte vernaculaire qu'il cite :

"te atua ka poi te fakafua, [...]; e tupu ki te uga" traduit par l'auteur: "l'esprit qui souhaite aller et

3. Paea-a-Avehe, né en 1889, fut l'un des principaux informateurs de Stimson. Originaire de Anaa, il a été formé à l'ancien culte et aux traditions anciennes par son oncle Tiapu, un "célèbre tahuga " (Stimson, 1933 : 66). Les informations délivrées par Paea concernant la cosmogonie et la religion ancienne ont parfois été mises en cause, notamment par Kenneth Emory dans son article "Tuamotuan concepts of creation " (Emory, 1940).

4. Il est à noter que ce type de jugement a souvent été appliqué aux figures divines ou ancestrales pré-chrétiennes par les autochtones eux-mêmes après leur conversion au christianisme. Voir à ce sujet Babadzan (1982).

5. Stimson renvoie aux termes : tagata, mahoi, rigorigo, korio, manuku, tupapaku, hoahoatua, maitu, mauri. 
transmettre la maladie se change en crabe. " (Firth, $1930: 304)$

\section{À Hawai'i, les kupua font partie :}

«d'une catégorie d'esprits qui sont [...] inférieurs en terme de statut aux dieux primordiaux " (Handy, $1968: 52)$,

le dictionnaire hawaïen d'Elbert et Pukui ajoute que ce sont des demi-dieux,

"des êtres surnaturels possédant plusieurs formes. " (Elbert et Pukui, 1986 : 141)

Ailleurs dans le Pacifique, tupua est synonyme d'esprit (Fidji, Tuvalu) ou de monstre (Rarotonga). La définition appartenant au dialecte de Sikaiana dans les Îles Salomon apporte un autre éclairage. Dans cette dernière, il est indiqué que le tupua est un

"esprit surnaturel mais contrairement aux aitu, [eux] ne sont pas des personnes mortes devenues des êtres surnaturels mais ont toujours été des êtres surnaturels. » (www.pollex.org.nz)

Ces différentes définitions rappellent l'opposition posée par Codrington et rappelée par Jean Guiart " entre les dieux qui ont été humains et ceux qui n'ont jamais été des humains ${ }^{6}$ " (Guiart, 2008 : 25) dans le Pacifique.

Le terme aitu serait, d'après la dernière définition, plus proche de l'entité que nous cherchons à décrire puisque les tuputupua appartiennent au rang des divinités inférieures, c'est-à-dire celles ayant eu une vie humaine, par opposition aux divinités de rang supérieur, communément nommées atua dans les langues polynésiennes. La proto-forme austronésienne de aitu, qaitu désigne l'esprit d'une personne décédée. Il en va de même pour le terme paumotu ${ }^{7}$ qui en est issu, maitu. Stimson, dans son dictionnaire, ajoute que cet esprit pouvait être invoqué par un taura, un terme sur lequel nous reviendrons, et que certains rites étaient nécessaires pour transformer un esprit normal (vaerua) en maitu. ${ }^{8}$ Un terme paumotu dérivé de maitu, hoahoamaitu, est défini par les informateurs de Stimson comme des «esprits qui ont pour habitude de prendre possession de corps humains; il s'agit des esprits des ancêtres, morts de vieillesse, que les anciens ont déifiés tels des dieux " (Stimson, 1933 : 27). $\mathrm{Si}$, l'entité prend ici possession du corps d'un homme et non pas d'un animal, son rôle, lui, peut être similaire puisque ils "peuvent être invoqués par leurs descendants et leurs proches afin de leur venir en aide» (Stimson, 1933 : 57).

Ailleurs aux Tuamotu, Paul Ottino, dans un développement consacré à la présence d'une éventuelle forme de totémisme dans l'archipel, emploie quant à lui le terme täura'. Dans Ethnohistoire de Rangiroa, il désigne donc un "tāura", comme étant un "symbole attaché à des personnes, groupes sociaux ou régions géographiques" (Ottino, 1965: 109). Ces symboles sont quasi-exclusivement des espèces animales. Le terme de tãura est également usité à Tahiti et dans les autres îles composant l'archipel de la Société, ce qui peut se comprendre étant donné la proximité linguistique qui existe entre la langue tahitienne et le dialecte Mibiroa parlé à Rangiroa. L'académie tahitienne le définit ainsi comme un " totem, un dieu tutélaire ».

Au final, pour la zone qui nous concerne, nous retiendrons le terme tuputupua à la fois car il est celui employé par les Paumotu lors des récits que nous étudierons mais aussi en raison de son étymologie qui nous semble s'accorder avec les principaux éléments de ces mêmes traditions. Par ailleurs, nous considérerons le terme tāura comme son équivalent dans la zone sous influence tahitienne (archipel de la Société et aire Mihiroa aux Tuamotu).

\section{État des connaissances sur le sujet aux Tuamotu}

Les croyances que nous allons étudier ont souvent été regroupées sous le terme "totémisme" quelle que soit la société étudiée. Or, les formes décrites sont parfois si variées qu'il convient de s'interroger sur le bien-fondé de cette dénomination. Lévi-Strauss écrivait à ce propos que «le prétendu totémisme échappe à tout effort de définition dans l'absolu " (Lévi-Strauss, 1962: 11). Partant de ce constat, il désignait quatre combinaisons possibles selon le type de relation observée entre l'homme (soit en tant qu'individu soit en tant que groupe de parenté), et l'animal (soit en tant qu'individu soit en tant qu'espèce). Nous pouvons résumer ainsi :

Au sujet de ces diverses combinaisons, LéviStrauss notait que seules "les deux premières ont été incluses dans l'aire du totémisme " (LéviStrauss, 1962 : 28), laissant donc notamment de côté la relation la plus courante en Polynésie.

6. Cette opposition se retrouve dans la terminologie paumotu avec les expressions nuku-mau-atua et nuku-mau-tagata. Stimson définit la seconde comme l'ensemble des dieux inférieurs, l'appellation tagata rappelant leur précédente humanité. Les tuputupua font vraisemblablement partie de cette catégorie.

7. Le terme Paumotu est le nom désignant la population de l'archipel des Tuamotu, il est aussi utilisé en tant qu'adjectif.

8. Ces rites consistaient notamment au prélèvement des reliques (ongles, cheveux...) du défunt en vue d'une conservation.

9. À ne pas confondre avec le terme taura (sans longueur sur le a), vu plus haut, qui est une personne capable d'invoquer une entité spirituelle. 


\begin{tabular}{|l|c|c|}
\hline & Type de relation & Exemple de zone concernée \\
\hline $1^{\mathrm{e}}$ catégorie & $\begin{array}{c}\text { Relation entre une espèce naturelle dans son } \\
\text { ensemble et un groupe de parenté }\end{array}$ & Australie \\
\hline $2^{\mathrm{e}}$ catégorie & $\begin{array}{c}\text { Relation entre une espèce animale et un } \\
\text { individu }\end{array}$ & Amérique du Nord \\
\hline $3^{\mathrm{e}}$ catégorie & $\begin{array}{c}\text { Relation entre un individu animal ou végétal } \\
\text { et un individu humain }\end{array}$ & Polynésie, Mélanésie \\
\hline $4^{\mathrm{e}}$ catégorie & $\begin{array}{c}\text { Relation entre un individu animal et un } \\
\text { groupe de parenté }\end{array}$ & \\
\hline
\end{tabular}

TABLEAU 1. - Les différents types de relation totémiques dans le monde (selon Lévi-Strauss, 1962 : 27-28)

Dans l'archipel des Tuamotu, Eugène Caillot et Paul Ottino sont parmi les rares auteurs à s'être intéressés à ce thème. Le premier a notamment consacré une partie de son ouvrage Histoire des religions de l'archipel Paumotu à ce qu'il nomme "la religion du totémisme». Dans celle-ci, Caillot indique que, selon lui, le totémisme était une religion très ancienne que les Paumotu avaient déjà délaissée à l'arrivée des Européens et dont il ne restait de ce fait que quelques survivances. Il en prend pour preuve le peu de sources qu'il a pu recueillir sur le sujet lors de ses visites (un seul récit provenant de l'île de Raroia ${ }^{10}$ ).

Ces remarques nous semblent aujourd'hui bien étonnantes tant cette croyance et les récits qui en relèvent sont encore assez bien connus, au contraire de ceux relatifs à la religion ancienne. La description que Caillot fait du "totémisme " paumotu s'accorde tout à fait avec les traditions que nous allons aborder. Nous citons :

"certains [des ancêtres] devenaient [...] des animaux ou des végétaux, surtout des animaux de terre ou de mer ; leur esprit passait alors dans le corps d'une bonite, d'un requin ou d'une raie, d'un crabe, d'un chien ou d'un porc, etc. Il en résultait que leurs descendants se croyaient ensuite apparentés à l'un de ces animaux-là et qu'ils le considéraient comme leur protecteur. Pour ce motif, ils s'abstenaient de le tuer et de le manger. " (Caillot, 1932 : 85)

Cette citation nous permet de présenter les principaux points de discussion au sujet des tuритириа. D'après les informateurs de Caillot, il s'agit donc d'ancêtres et non de dieux (atua) qui prennent forme animale, et plus rarement végétale. L'esprit de l'ancêtre prend possession d'un animal, il n'est donc pas question d'une transformation physique d'humain à animal. Les espèces concernées sont variées et sont tout aussi bien terrestres que marines. La description de Caillot tendrait à considérer le "totémisme " paumotu comme relevant de la quatrième catégorie de relation homme-animal suivant la classification vue plus haut, une relation entre un animal isolé et un groupe de parenté. Toutefois, le manque de précision de son propos ("l'un de ces animauxlà ») maintient un doute. La relation établie pourrait ainsi tout à fait être de type groupe de parenté - espèce animale. Nous aurons l'occasion de revenir sur ces différents points et de discuter leur validité au regard des traditions que nous avons recueillies.

Paul Ottino, de son côté, est allé plus loin dans l'analyse, en distinguant trois catégories distinctes de tāura (Ottino, 1965 : 110). La première catégorie comporte ceux qu'il estime commun à une région ou à une île, il les nomme "tāura généraux ». Ils ont pour particularité d'avoir un nom qui leur est propre indiquant par là qu'il s'agit d'individus particuliers. La deuxième catégorie est celle des " tãura d'ancêtres », ils sont rattachés à un ancêtre et à ses descendants et possèdent en plus d'un nom, une résidence qui est connue (un marae, un récif, etc.) ce qui les distinguent de la troisième catégorie, les "tãura des 'ati " qui n'ont ni localisation géographique précise, ni nom et correspondent ainsi à des espèces prises dans leur ensemble. La situation décrite par Ottino est plus complexe que celle présentée par Caillot puisqu'on retrouve trois des quatre combinaisons évoquées plus haut dans notre tableau. Les "tãura généraux " correspondent à la quatrième catégorie, celle que l'on retrouve majoritairement en Polynésie et Mélanésie, c’est à dire une relation entre un groupe humain et un animal particulier. Les "tāura d'ancêtres" appartiennent à la fois à la troisième catégorie (relation entre un individu humain et un animal particulier) et à la quatrième catégorie (car il est transmis aux descendants et donc au groupe de parenté), tandis que les «tãura des 'ati » rejoignent la première catégorie.

Enfin, selon Éric Conte, sur l'atoll de Napuka, on dit des tuputupua qu'ils " étaient réservés aux souverains qui en héritaient de leurs parents " (Conte, 1988 : 162). L'auteur note toutefois l'existence de tuputupua " ayant appartenu à des individus, des familles ou même à des groupes

10. L'explorateur français Eugène Caillot voyagea dans les Tuamotu en 1900 et en 1912. Il visita principalement des atolls de la zone Tapuhoe (Makemo, Hikueru, Raroia, etc.). 
de familles, en dehors de la lignée des souverains " mais précise que ces récits sont récents, ce qui pourrait révéler une " conception tardive " (Conte, 1988 : 162) de cette croyance.

\section{Traditions relatives aux tиритириа dans l'archipel des Tuamotu}

\section{Turia, l'ancêtre requin (Makemo) \\ "E purehuga au na Vahua, te vahine a Korofakata. Vahua, ariki vahine, hakamanahia na Mahia. Kua putarihia vau na ta matou tuputupua, te paraoa e te mago ".}

" Je suis un descendant de Vahua, la femme qui s'est liée à Korofakata. Vahua, la ariki (chef), qui a obtenu son pouvoir de Mahia [ancêtre fondatrice du gati ${ }^{11}$ Mahia de Makemo]. Je suis capable de guider nos tиритириа, la baleine et le requin. "

Extrait d'un puta tupuna ${ }^{12}$ de Makemo [date inconnue]

À Makemo, l'existence de tuputupua sous forme de requin nous a été confirmée par Tina Apuarii, née Mamatui. C'est elle qui nous livre la première tradition de notre étude. Ce récit nous a été présenté comme une histoire familiale. Contrairement à l'extrait de puta tupuna cité, il ne semble pas être lié à un gati dans son ensemble, bien que les généalogies permettent de rattacher cette famille au même gati, le gati Mahia. Le récit se situe à une époque récente, la protagoniste principale, Toroatua, grand-mère de Tina, ayant vécu au $\mathrm{Xx}^{\mathrm{e}}$ siècle (1910-1988).

« La sœur de ma mère, Mama Tipapa, se faisait tout le temps battre par son täne [mari]. Elle a fui le village de Raraka en pirogue. Elle a dérivé pendant trois jours sans manger et sans boire d'eau. Ses enfants l'ont cherchée pendant deux jours sur l'île et en mer. Sans résultats. Alors les enfants ont averti ma grand-mère Toroatua Maifano, qui habitait Makemo, que sa fille avait disparu et qu'elle était introuvable. Alors, Toroatua est partie à tua [côté océan] pour prier Turia, notre tиритириа qui est un grand requin. Turia est un de nos ancêtres, il est mort en mer et a été mangé par les requins. Il est devenu un requin à son tour et est apparu en rêve à sa famille après sa mort afin de prévenir qu'il était mort et qu'il était devenu un requin.

Toroatua avait la réputation d'être une "sorcière"13. Elle a fait un pehepehe [chant] en l'honneur de Turia. Après son rituel, elle était persuadée que sa fille était encore vivante comme si Turia le lui avait dit.

Tipapa était très faible sur sa pirogue et si elle avait fui, c'était pour mettre fin à ses jours. Elle a témoigné par la suite que sa pirogue avait été soulevée par un grand poisson. Elle ne voyait ni l'avant, ni l'arrière.
Lorsqu'elle est arrivée sur l'île de Raraka, elle a vu la queue du requin partir au loin. Elle a donc raconté aux villageois et à sa famille que c'était Turia qui était venu à son secours.

Le lendemain Toroatua a été avertie que sa fille était saine et sauve. Elle remercia Turia avec un nouveau pehepehe."

Le tuритириа porte ici un nom, celui de l'ancêtre familial disparu en $\operatorname{mer}^{14}$, il renvoie donc à une créature unique et non à l'ensemble des requins. Nous sommes ici dans la catégorie de relation homme - animal la plus connue en Polynésie et Mélanésie ( $c f$. catégorie 4 du tableau). Un des éléments les plus intéressants de ce récit est la description de la manière par laquelle l'ancêtre s'est mué en requin puisque c'est en ayant été ingéré par ces derniers, après sa mort, que Turia est devenu l'un d'eux. Nous pouvons penser, bien que cela ne soit pas énoncé dans le texte, qu'il s'agit en fait de l'" esprit » du défunt qui a pris possession du corps d'un requin. Un récit semblable, recueilli par Caillot à Bora-Bora, intitulé "Te mau ma'o tupapa’u", parle également d'un homme qui se laissant mourir

"demanda aux siens de le placer dans un bassin naturel de la rivière qui se jette dans la baie [...] au lieu de l'enterrer. Quelque temps plus tard, l'âme de ce jeune homme s'enferma dans le corps d'un requin, qui devint lui aussi un revenant. » (Caillot, 2008 : 133)

L'utilisation de termes comme ceux d' " esprit " ou d' " âme " n'est pas tout à fait pertinente dans le contexte océanien. Jean Guiart rappelle ainsi à juste titre qu'il conviendrait de privilégier la notion de "souffle" pour discuter de telles croyances locales. Cette notion pourrait être traduite en paumotu par " iho " que Stimson définit comme le moi-intérieur, une essence qui réside dans toutes les créatures vivantes (Stimson et Marshall, 2008 : 174). Jean Guiart écrit :

"ainsi le fil de l'existence n'est-il pas brisé par la mort, cette dernière provoquant seulement un changement de support de l'être toujours vivant. " (Guiart, 2012 : 10)

C'est bien cette conception de l'au-delà qui est décrite dans notre récit.

Une fois devenu requin, Turia, avertit ses proches en leur apparaissant en rêve. Le récit ne nous dit pas qu'elle était sa forme à ce momentlà, humaine ou animale, mais l'apparition d'un défunt en rêve afin de communiquer avec son

11. 'Ati en tahitien. Il s'agit d'« un groupe social basé sur la descendance en ligne directe à partir de l'ancêtre dont le groupe porte le nom " (Stimson et Marssall, 2008 : 93).

12. Livre rédigé et conservé par les anciens.

13. Dans le vocabulaire actuel, notre interlocutrice serait tentée de donner comme équivalent au terme "sorcière " qu'elle a employé, celui de "tagata manamana" littéralement une personne aux pouvoirs magiques.

14. D’après les généalogies, Turia aurait vécu quatre générations avant Toroatua, c'est-à-dire vers l'an 1800. 
cercle familial est un phénomène connu et plusieurs fois décrit par les anthropologues océaniens. Par exemple aux îles Salomon, Guiart décrit un rituel qui comporte de nombreux points communs avec notre récit :

« il est fréquent que les morts soient apportés en offrande aux requins protecteurs, le symbole étant que ces morts allaient se transformer eux aussi, en requins protecteurs. La communication avec eux est par les rêves divinatoires, ceux des membres du groupe de descendance intéressé » (Guiart, 2012 : 41).

Beckwith relève une tradition à Hawai'i où un enfant avorté est mis en terre par ses parents mais un aumakua

"apparut en rêve et leur dit que ce n'était pas bien, alors ils le jetèrent à la mer et il devint un requin. " (Beckwith, 1917 : 504)

Nous verrons plus loin que les aumakua de Hawaii sont une entité particulièrement proche des tиритириа.

Une des grandes interrogations concernant les tuputupua est de savoir s'ils sont liés ou non à une forme de culte, un culte qui pourrait être compris ou indépendant du culte des ancêtres. Le récit nous donne à ce sujet quelques éléments de réponse.

Les actions effectuées par Toroatua sont en effet qualifiées de rituelles, celle-ci semble posséder des capacités afin d'entrer en contact avec le tuputupua qui ne sont pas communes, comme le prouve l'appellation de "sorcière " qui pourrait correspondre au terme "taura" tel que défini par Stimson plus haut. Dans les temps anciens, on constatait effectivement que l'invocation des ancêtres était seulement réservée à certaines personnes pourvues de $m a n a^{15}$. Le détail du rituel, lui, n'est pas clairement évoqué, si ce n'est l'usage de pehepehe, c'est-à-dire un chant ou une récitation, le premier servant à invoquer le tuputupua, le deuxième à le remercier d'avoir répondu à l'appel. Cette relation avec les ancêtres a été commentée par Caillot qui écrit que :

" on ne les honorait que passagèrement et d'une manière intéressée, lorsqu'on avait à réclamer leur service. » (Caillot, 1932 : 68)

Cette subordination de l'ancêtre au profit des vivants a été également abordée par Martha Beckwith qui écrivait que les aumakua

« sont liés par obéissance à ceux qui les vénèrent et qui deviennent leurs gardiens. » (Beckwith, 1917 : 503)
Cette citation suggère une réciprocité entre l'homme et l'animal qui se protègent mutuellement.

Un autre élément laissant penser à un rituel dans notre tradition est le lieu de l'invocation, puisque Toroatua semble se rendre à un endroit précis côté océan. Il s'agit, selon notre interlocutrice, de la terre Mama-hua-i-ragi. Cette terre, dominant l'océan, constitue le point le plus élevé du village de Pouheva à Makemo. Une interprétation pragmatique voudrait qu'elle s'y rende afin d'obtenir une plus grande proximité avec le lieu de résidence de Turia, le grand océan.

En marge du récit lui-même, une autre forme de communication entre Turia et Toroatua, moins ritualisée et plus spontanée, a été détaillée par notre interlocutrice. Fréquemment, lorsque Toroatua se baignait dans le lagon, elle ouvrait son pareo afin de recueillir Turia qui avait alors la forme d'un jeune requin. Ce dernier se mettait à tourner autour d'elle comme pour s'enrouler dans son pareo. Elle était capable d'échanger avec lui. Ainsi après l'avoir enjoint à partir, il s'éloignait d'elle tout en grandissant au fur et à mesure de son parcours pour finalement atteindre une taille démesurée. Cette scène fait écho à une anecdote relevée par Anne Hervé ${ }^{16}$ dans Magie et sorcellerie aux Tuamotu. L'auteur raconte comment, à Hikueru, une femme :

" s'était soigneusement lavé les cheveux, baignée à l'eau douce, frottée de monoï [huile de coco parfumée]. Revêtue d'une robe fraîche, assise à l'arrière de la pirogue, elle s'était fait conduire par son fils, alors enfant, à la recherche du banc de komene [chinchards] signalé. L'ayant trouvé, elle fit arrêter la course et harangua les poissons de loin, en très vieux langage. Puis la femme se rapprocha du banc de poissons sans l'effaroucher et fit décrire des cercles autour de lui, jusqu'à ce que les poissons tournent aussi en rond, se missent à la suivre. " (Hervé, 1948 : 53)

Ces deux anecdotes permettent de nuancer les affirmations maintes fois répétées de l'interdit pour la femme d'interagir avec le milieu marin, ainsi que celles affirmant que la possession du mana est fondamentalement masculine ${ }^{17}$.

Ce récit permet également d'appréhender le rôle dévolu aux tuритириа et les raisons qui amènent à leur invocation. Ce rôle consiste à porter secours à leurs descendants en danger. Ici, Turia doit empêcher la perdition en mer de Tipapa. Ottino relate des faits quasi-similaires à Rangiroa où le « requin-ancêtre " Avirimata'i, vivant dans un courant entre Rangiroa et Kaukura,

15. «Une puissance ou potentialité extra-physique, dérivée de la nature ou de la magie, et qui est inhérente à, ou émane de la nature " (Stimson et Marshall, $2008: 280$ ).

16. Fille de l'administrateur des Tuamotu, François Hervé, ayant passé les vingt premières années de sa vie dans l'archipel au début du $\mathrm{Xx}^{\mathrm{e}}$ siècle.

17. Voir notamment Handy cité par Gunson : «les environs du rituel [...] étaient fermés aux femmes "car elles étaient ordinaires (sans mana)" "(Gunson, 1982:335). 
«peut se manifester aux occupants d'une embarcation perdue en mer et leur indiquer en nageant droit devant eux la direction d'une terre. » (Ottino, 1965 : 115)

Cette croyance de l'intervention de l'ancêtre sous forme de requin afin de servir de guide a su traverser les époques. En 1976, le marin paumotu de la fameuse pirogue Hokule'a, Rodo Toku Williams, confie à l'anthropologue Ben Finney que les requins qu'ils aperçoivent

" sont ses esprits gardiens venus des Tuamotu. Les squales guident la pirogue vers les eaux natales du navigateur. " (Dunis, 1990 : 39)

Si, dans notre récit, Turia sert effectivement de guide, son rôle est plus important encore puisque c'est lui qui "soulève " la pirogue prenant alors une dimension héroïque. Cet aspect du récit rappelle une tradition recueillie dans le sud-ouest de la Nouvelle-Calédonie par Jean Guiart :

" une femme Tèè Kurëe étant partie à la pêche avec un Potyo [nom d'une famille], leur pirogue se mit à dériver pendant la nuit. L'homme réussit à rattraper la pirogue à la nage : il avait pris sur lui ses "herbes" liées au requin, mis son plumet dans les cheveux et appelé à son aide les requins tutélaires, les morts dont il était le descendant. Alors qu'il était en train de nager, l'un d'eux vint le soutenir sous l'aisselle. " (Guiart, 2015 : 65)

\section{À Tahiti, Teuira Henry note, quant à elle, que :}

« les requins possédés par les esprits de personnes mortes ne faisaient jamais aucun mal aux membres de leur famille lorsqu'ils les rencontraient en mer, bien au contraire, ils les secouraient en les portant sur leur dos ou entre leur mâchoires, jusqu'à terre. " (Henry, $1988: 400)$

Cette idée de requin protecteur a inspiré certains auteurs occidentaux ayant noté le lien étroit que pouvait entretenir les Paumotu avec cet animal. La confiance avec laquelle les gens de l'archipel se baignaient à proximité des squales et l'apparente absence d'animosité de ces derniers ont fait dire à Anne Hervé que :

"dans chaque atoll, les familles ont une sorte de pacte avec les requins copropriétaires; les hommes plongent, les femmes pêchent, les enfants se baignent sans crainte. " (Hervé, 1948 : 54)

Ce constat a souvent étonné les observateurs européens pour qui le requin inspirait plutôt de la crainte, mais nous ne croyons pas que ce type de communication systématique entre les habitants de l'archipel et l'animal en question ait existé. Ceci a probablement été imaginé par les
Européens afin de répondre à une situation jugée irrationnelle.

Le requin est toutefois un animal privilégié dans cette relation d'affinité que nous observons, aussi bien aux Tuamotu qu'ailleurs dans le Pacifique. Les traditions Hawai'iennes sont ainsi particulièrement riches en récits de ce genre, Beckwith indique qu'à Hawai' $i$

«sur la côte, les requins sont particulièrement l'objet de vénération. » (Beckwith, 1917 : 503)

Pour compléter notre analyse, il serait intéressant de déterminer si cette relation entre un groupe social et son animal tutélaire, en l'occurrence le requin, est associée à une interdiction de sa capture ou de sa pêche et de sa consommation. Pour ce qui est de Makemo, nous pouvons noter que pour les habitants actuels, le requin ne semble pas avoir été pêché. Il ne l'est pas de nos jours, et pour les personnes interrogées ne l'étaient pas non plus il y a quelques générations et ce, par quelle que famille que ce soit. Il est pourtant pêché dans l'ensemble de l'Océanie selon une technique relativement uniforme dite du nœud coulant. Aux Tuamotu, Paea-a-Avehe

« indiquait que certains grands requins étaient capturés en mer par un grand collet passé autour de la tête quand ils étaient à proximité d'une pirogue. " (Torrente, $2012: 94$ )

\section{Le kaveu de Raroia}

Le seul récit relatif aux tиритириа recueilli par Caillot est originaire de l'atoll de Raroia, au nord-est de Makemo. Il a été recueilli en 1912. Malheureusement, comme souvent chez Caillot, le nom de son interlocuteur n'est pas précisé, ce qui fait qu'il est compliqué de le rattacher à une famille ou un gati particulier de l'île. Ce récit met en scène un kaveu, ou crabe de cocotier.

"À Raroia, il y a un kaveu qui est l'ancêtre d'une certaine famille de là. Ce crabe vient se promener dans la maison de la famille, se repose, et quand il lance un certain cri, c'est que l'on dit du mal de cette famille-là. Et souvent il arrive que ceux qui en disent du mal se trouvent pris subitement de grandes coliques, leur ventre enfle, et l'on est obligé d'avoir recours immédiatement à la famille de qui l'on a dit du mal, pour qu'elle vienne frictionner le ventre du malade avec une certaine huile préparée exprès, et aussitôt le ventre désenfle, le malade est guéri. » (Caillot, 1932 : 86-87)

Dans ce récit, le rapport à l'ancêtre est clairement énoncé, ce kaveu est habité par le "souffle" d'un ancêtre ${ }^{18}$. La relation est de même nature que celle à Makemo entre la famille Maifano et

18. Il est qualifié de tupuna dans la version paumotu de la tradition. 
le requin Turia, c'est-à-dire une relation entre un groupe de parenté et un animal particulier, ce qui pourrait donc être la norme dans l'archipel.

Le kaveu n'est pas nommé, pourtant il est bien précisé qu'il s'agit d'un individu unique, ce qui irait à l'encontre des conclusions d'Ottino qui écrivait que seuls les tuputupua qui étaient considérés en tant qu'espèces ne portaient pas de noms (Ottino, 1965 : 111). Nous pouvons aussi penser que son nom n'a tout simplement pas été communiqué à Caillot par son interlocuteur.

La localisation du tuputupua est indiquée, il demeure autour et dans la maison familiale, entretenant ainsi sa proximité avec les vivants. Il est capable de communiquer avec eux grâce à son cri, son invocation n'est donc pas nécessaire. Cette aptitude, relevant de l'anthropomorphisme ${ }^{19}$, rappelle la présence de l'ancêtre dans le corps du kaveu.

Il joue comme les autres tuритирua un rôle de protecteur, en avertissant son groupe de descendance d'un danger, ici les commérages et les intrigues qui se trament à leur insu et qui pourraient les nuire. L'ancêtre kaveu démontre alors son pouvoir de nuisance, il n'est plus comme Turia un sauveur mais une entité capable de causer du tort à ceux qui se sont attaqués à sa famille. Ses actions ressemblent fort à un procédé relevant du muki (sorcellerie). Les douleurs ressenties par la personne visée par ce sort auraient pour origine la pénétration du tиритириа dans le corps de cette dernière, provoquant ainsi un gonflement. Anne Hervé écrivait à ce sujet :

«j'ai entendu des menaces verbales comme: "ton ventre va enfler; il y aura un crabe dedans, et tu mourras dans six mois".» (Hervé, 1948 : 49)

Cette anecdote démontre le rôle singulier du crustacé dans cette entreprise.

De tels procédés sont décrits par Teuira Henry, pour qui les tahutahu $u^{20}$ cherchaient à faire pénétrer dans les $t i i^{21}$ :

" de méchants esprits dont la réputation était encore connue ; on les appelait 'oromatua-'ai-aru ou 'oromatua-niho-roroa, [ils] étaient prompts à répondre à l'appel $[\ldots]$ pour aller faire du mal à des personnes qui leur étaient indiquées [...] Lorsqu'ils s'introduisaient dans une personne, les symptômes du pifao étaient caractéristiques; la victime souffrait d'horribles douleurs dans les intestins. » (Henry, 1988 : 212-213)

Teuira Henry emploie le terme de "'oromatua» pour qualifier les « esprits » utilisés pour pratiquer le pifao (terme équivalent au muki paumotu), or "'oromatua" est le nom qu'elle utilise pour désigner des entités semblables aux tuputupua à Tahiti :
"Les âmes [des morts] devenaient des Dieux inférieurs appelés 'oromatua qui se divisaient en trois catégories : 'oromatua maitatai qui réintégraient leurs propres crânes en tant que Dieux familiaux ou esprits gardiens ; 'oromatua 'oriori noa qui, de temps à autre apparaissaient aux membres de leur famille pour les conseiller ou les prévenir des dangers à venir ; et enfin les óromatua nihoniho roroa [...], ils étranglaient et dévoraient lorsqu'ils étaient contrariés." (Henry, $1988: 212-213$ ).

Le récit de Raroia se rapproche des observations de Raymond Firth à Tikopia qui écrivait au sujet de deux variétés de crabes que :

"à certains moments, on pensait qu'ils étaient pénétrés par des esprits itinérants, d'un genre malfaisant et porteur de maladie, envers lesquels l'homme devait en permanence être sur ses gardes. » (Firth, 1930 : 301)

Un autre tuputupua apparaissant sous la forme d'un kaveu nous a été présenté sur l'atoll de $\mathrm{Ni}$ hiru, situé à une cinquantaine de kilomètres à l'est de Makemo. D'après notre interlocuteur, Tukai Mairoto, un marae témoignerait de son existence. Lors de notre visite sur l'île, ce " marae kaveu ", terme employé par Tukai, s'apparente à une ciste surmontée d'une pierre sur laquelle est gravée le nom de l'ancêtre, Teuru (ou Tearu) a Painara, à la manière d'une pierre tombale. Cette inscription démontre l'aspect contemporain de cette croyance. L'influence de ce tupututua s'exerce à la fois sur Nihiru et sur Marutea Nord en raison de liens généalogiques entre les familles occupant encore ces deux atolls. Ainsi à Marutea, la capture du tuритириа par une personne qui n'aurait pas été capable de le reconnaître aurait pour conséquence l'apparition soudaine d'une multitude de kaveu visant à assaillir l'auteur d'une telle imprudence.

\section{Matirohe, l'homme-murène de Nibiru}

À Nihiru, nous avons recueilli un autre récit relatif aux tuрutupua. Il nous a été transmis oralement par Loulou Mairoto, né sur l'atoll en 1954.

"One-roa est le motu [îlot] sur lequel se déroule l'histoire de Matirohe. Matirohe est une créature mihomme, mi-murène. Sept hommes seraient venus en pirogue jusqu'à Nihiru, ils sont venus à One-roa depuis une autre île qui pourrait être Mangareva. Ces hommes étaient affamés après leur périple. Ils ont alors capturé une murène qui se trouvait être Matirohe. Ils ont préparé un ahima'a [four en terre traditionnel] pour leur repas, ils ont préparé la murène mais ont oublié la tête. Ils se sont reposés mais la murène s'était reformée. Le lendemain, alors qu’ils reprirent la mer à marée haute, la murène, cachée sous la pirogue,

19. Le seul son qu'émet le crabe de cocotier est un claquement produit lorsqu'il se déplace.

20. Tahutahu : sorcier, jeteur de sort (Fare Vanáa).

21. Tï : image d'un Dieu, statue. Mauvais esprit utilisé par les tahu'a nanati pour exécuter les maléfices (Fare Vana’a). 
les tua un par un puis renvoya la pirogue hors de $\mathrm{Ni}$ hiru. Matirohe repartit alors à One-roa sur le marae 22 Patita. Ce marae se trouve côté océan.

On dit que Matirohe est le gardien, le protecteur des habitants de Nihiru. Ainsi Tutere Tehiva, le catéchèse de l'île, fit appel à Matirohe lorsque son mât se brisa entre Taenga et Nihiru. Il fut sauvé par Matirohe. »

Contrairement à Turia de Makemo, le nom de Matirohe est connu en dehors de Nihiru. Stimson écrit à son propos qu'il s'agit du nom d'un "démon du monde de la nuit, ayant le corps d'une murène" (Stimson et Marshall, 2008 : 295) et l'a recensé sur l'île de Hao. Le dictionnaire mythologique de Robert Craig comprend Matirohe dans sa liste des "eels deities" (Craig, 1989: 332). D'après lui, cette divinité est commune à l'archipel des Tuamotu. Paul Ottino confirme cette hypothèse dans son étude puisqu'il fait de Matirohe (orthographiée Matiore) un "tāura général " suivant sa classification. Matirohe serait ainsi d'après lui une murène noire liée à l'ensemble des Tuamotu de l'ouest. Un de ses interlocuteurs donne de plus amples informations :

" il y a très longtemps alors qu'il n' existait pas de 'ati [...] il existait des tāura qui, dans le Mihiroa, étaient une petite murène noire appelée Matiore et un crabe de cocotier (kaveu) Heiau. [...] Matiore était douée d'une force prodigieuse. » (Ottino, 1965 : 112)

Plus loin, il ajoute que Heiau et Matirohe étaient les "vrais dieux" des Tuamotu dans les temps anciens et que :

«[ils] inspirent une crainte qui diffère de l'attitude quasi indifférente à l'égard des autres symboles. " (Ottino, $1965: 116$ )

Matirohe serait donc une entité particulièrement ancienne, voire primordiale, et connue au moins dans la zone Tapuhoe et Mihiroa. La force qu'on lui prétend et la crainte qu'elle inspire semble justifiée au regard du récit que nous avons recueilli. Le nom Matirohe est recensé par le site fishbase.org comme étant le nom paumotu donné au saddled snake eel (pas de nom français) de la famille des anguille-serpents. Elle se rapproche beaucoup de l'anguille-serpent annelée qui est :

" [une] espèce mimétique du fameux tricot rayé, véritable serpent de mer, avec lequel elle est fréquemment confondue. " (Bacchet et al., 2006 : 111)

\section{Elle est jugée inoffensive.}

Dans ce récit, nous n'avons pas de preuve de l'ancestralité de Matirohe, ce qui fait qu'il est impossible de le rattacher à un quelconque groupe social. Le type de relation paraît être le même que celui observé précédemment entre une créature animale unique et un groupe de parenté, qui semble être particulièrement étendu dans ce cas précis, puisqu'il est dit protéger l'île toute entière face à d'éventuelles menaces extérieures. Cette menace prend la forme d'une embarcation venue de Mangareva, dans l'archipel des Gambier, une origine lointaine représentant un danger pour la population de l'île. En effet :

« lors de la venue d'une pirogue étrangère sur un atoll, la coutume voulait que les partis en présence déclament leur généalogie [...], soit les deux groupes en présence se trouvaient des ancêtres communs et il était alors de règle que les arrivants soient accueillis pacifiquement [...], soit les noms énumérés ne comprenaient aucun lien de parenté et les gens de l'atoll en concluaient sur le champ que les arrivants venaient avec des intentions belliqueuses. » (Torrente, 2012 : 297)

Bien que des liens aient pu exister entre les Tuamotu et les Gambier à l'époque pré-européenne dans laquelle semble se situer notre tradition (cf. la référence au marae), une telle arrivée a certainement été perçue comme de mauvaise augure. D'autant qu'en s'attaquant à l'esprit protecteur de l'île, ces hommes ont prouvé leur ignorance des croyances locales.

Un autre élément spécifique à ce récit nous intéresse, il s'agit du lien qui est fait entre le tuputupua et un marae particulier, le marae Patita. Le motu One-roa sur lequel se trouve ce marae est le théâtre des événements qui sont narrés. Il est situé au nord-ouest de Nihiru. D'après notre interlocuteur, le marae Patita est situé à l'extrémité ouest de l'îlot, côté océan. Un marae a été relevé sur ce motu lors de la mission archéologique du CPSH menée par Éric Conte en 1990. L'auteur le désigne sous le nom de marae Nuhiva (Conte, 1990 : 75). S'il s'agissait du même marae, ses dimensions apparemment imposantes (20 mètres sur 15 d'après l'informateur d'Éric Conte) laissent à penser qu'il s'agissait d'un marae d'importance sur l'île ${ }^{23}$. Nous pouvons déduire au vu du récit que Matirohe est le gardien du marae Patita. Ceci rappelle par exemple le marae Taputuarai de Papara qui :

« avait comme gardien tutélaire le dieu Mo’o qui se manifestait sous la forme d'un lézard. ${ }^{24}$ »

22. "Enceinte sacrée [...] destinée aux dieux " (Torrente, 2012 : 246).

23. Il est à noter que l'ancien village de Nihiru se situait sur le motu One-roa. C'était également à l'époque pré-européenne, la principale zone d'occupation. Ainsi la place d'assemblée de la chefferie portait le nom de ce motu, One-roa (Henry, $1988: 115$ ).

24. Article sur : www.tahitiheritage.pf/lezard-marae-papara/ citant notamment Kenneth Emory, Marau Taaroa et Raymond Graffe comme sources. 
À noter que la présence d'un tuритириа sur un marae est indiqué par un bruit particulier, le poko, qui est " le son de crépitement du feu " (Stimson et Marshall, 2008 : 399). Stimson semble néanmoins faire ici référence à une présence qui n’est pas nécessairement d'ordre physique.

Au cours de la narration, nous apprenons qu'outre le non-respect du tapu qui pèse sur Matirohe, les hommes de Mangareva commettent une seconde erreur, celle de le manger en omettant de consommer sa tête. Or, comme nous allons le voir dans un passage du cycle du guerrier Honokura qui rejoint ce récit, la tête de l'animal est l'endroit où se situe le «souffle" de l'ancêtre. Pour tuer le tиритириа, il est donc nécessaire de porter le coup fatal au niveau de cette partie du corps. L'erreur des hommes de Mangareva entraînera la régénérescence de Matirohe. Une créature qui, par ailleurs, possède une autre particularité que n'ont pas les autres tиритириа que nous avons décrits, la capacité de passer à sa guise d'une forme à une autre, puisqu'il semble capable de se transformer indéfiniment en homme ou en murène.

Ces éléments évoquent un passage du cycle de Honokura dans lequel ce dernier cherche à venger Tuiha'a, jeune chef de son district assassiné par des guerriers venus des Tuamotu. Le corps de Tuiha'a ayant été amené sur un marae de Takume, Honokura décide de s'y rendre avec ses guerriers. Takume est alors défendu par le guerrier Te-akuroa, que l'on peut traduire littéralement par « le grand espadon ". Ce dernier est, dans le récit, tantôt présenté comme un kaito (guerrier), tantôt comme un "dieu marin protecteur" sous forme d'espadon (Henry, 1988 : 543). Par le biais d'une ruse, Honokura attire Te-aku-roa jusqu’à lui, la tradition dit alors que :

« les frères de Honokura voulaient couper la queue du poisson, mais celui-ci leur conseilla de n'en rien faire car l'esprit se tenait dans la tête. " (Henry, 1988 : 543)

La suite du récit est particulièrement intéressante :

"le monstre ayant reculé dans la mer prit son élan et d'un coup de queue formidable se précipita comme une flèche sur Honokura pour le percer de part en part. Ce dernier, qui l'attendait de pied ferme, lui enfonça sa lance dans la bouche et le tua net. Pour empêcher l'esprit démon ${ }^{25}$ de demeurer dans son incarnation, Honokura découpa le poisson avec sa hache magique appelée Te-marua. Les morceaux enveloppés dans des feuilles de cocotier, furent mis au four et mangés tandis que le prêtre envoyait le mauvais esprit chez les démons. » (Henry, 1988 : 544)

Dans cette tradition, Te-aku-roa a le même rôle que Matirohe, défendre son île face à une menace extérieure. Comme l'homme-murène de Nihiru, il peut se transformer en espadon puis revenir à une forme humaine. Un rituel est mis en place après sa mort. Il a pour but d'extraire l'" esprit " (ou plutôt le souffle, "iho ») de l'ancêtre de son incarnation animale et de le guider au Pō, dernière demeure de ce souffle après la mort. Ce parcours post-mortem est bien connu dans le triangle polynésien. Ainsi, un observateur comme Caillot pouvait noter que pour les Paumotu :

"l'âme n'était que le souffle qui sort du nez et de la bouche d'un individu, elle devenait après la mort de celui-ci, un esprit [...]. Après sa séparation d'avec le corps, l'esprit entreprenait un voyage lointain, durant lequel il devait renverser des obstacles de toute nature, avant de parvenir à sa nouvelle et dernière résidence. " (Caillot, 1932 : 31)

Cette variante de Takume confirme une nouvelle fois l'hypothèse émise en amont: l'ancêtre ne se transforme pas en animal mais c'est son souffle qui prend possession d'un corps animal. Cet esprit réside dans la tête de ce dernier, c'est pourquoi Honokura prend bien garde de ne pas commettre la même erreur que les assaillants de Matirohe dans la tradition précédente, et de consommer l'espadon en entier. La mise à mort et la consommation de Te-aku-roa entraîne le courroux des habitants de Takume mais également, d'après la tradition, de l'ensemble des habitants de l'ouest de l'archipel. Ceci peut être interprété de deux façons qui ne sont pas incompatibles, soit Te-aku-roa est un tuputupua général comme les « tãura généraux " présentés par Ottino, c'està-dire qu'il est connu et invoqué dans une zone étendue, en l'occurrence les Tuamotu de l'ouest, soit ce ralliement prouve que Takume entretenait des liens particulièrement forts avec ces îles. En effet, le gati Varoa, dont est issue la cheffesse de Takume, Maruia, exerçait une influence importante dans l'archipel. De nombreux gati installés dans les îles de la zone sont issus du gati Varoa.

\section{Bilan de l'étude et comparaison avec les autres sociétés du Pacifique}

\section{La nature de l'entité tuputupua}

D'après les quelques variantes que nous venons d'étudier, un tuputupua pourrait être défini comme la réincarnation d'un ancêtre dans un corps animal après que le iho de celui-ci ait pris possession du corps en question. Frédéric Torrente décrit, en effet, le iho comme une 
«essence vitale circulant de façon continue dans chaque individu ou chose vivante. " (Torrente, 2012 : 113)

Cette définition pourrait s'appliquer à l'ensemble des récits étudiés, bien que le rapport à un ancêtre ou la manière par laquelle l'esprit se fond dans l'animal ne soient pas parfois évoqués. $\mathrm{Au}$ sujet des espèces animales concernées, nous en avons recensé douze aux Tuamotu : le requin, la baleine, le crabe de cocotier, l'espadon, le chien, la murène, le pluvier fauve, le noddi brun, le poisson-flûte, le crabe, le poulpe et le cochon. Nul doute que cette liste viendrait à s'allonger en cas d'étude extensive sur le sujet. On remarquera tout de même l'absence de la tortue qui, dans l'archipel, peut être considérée comme l'animal sacré par excellence. Cette relative disparité dans le choix des espèces fait penser aux remarques de Meyer Fortes, citées par Lévi-Strauss :

«les animaux totémiques des Tallensi [tribu du Ghana] ne forment donc une classe, ni au sens zoologique, ni au sens utilitaire, ni au sens magique. Tout ce qu'on peut en dire est qu'en général, ils appartiennent à des espèces sauvages ou domestiques assez communes. » (Lévi-Strauss, 1962 : 109)

Le principal point de convergence entre les traditions étudiées est le rôle dévolu au tuputupua qui devient le protecteur du groupe de parenté dont il est issu. Il le protège d'éventuels envahisseurs, de dangers divers telle une dérive maritime ou l'influence néfaste de groupes sociaux concurrents. En revanche, contrairement aux affirmations de Paul Ottino, rien ne prouve que leur rôle ait pu être plus important jusqu'à représenter de manière symbolique le groupe de parenté auquel ils sont rattachés. C'est sans doute ce qui constitue une des principales différences avec le totémisme nord-américain.

Avant de tirer toute conclusion, il convient néanmoins de prendre en compte la question de l'évolution du sens de tuputupua au fil du temps. Nous avons vu que les informateurs de Stimson faisaient entrer, dans la catégorie des tuритириа, une grande partie des créatures du Pō pouvant entrer en interaction avec les vivants ou avec les « esprits » de ceux venant tout juste de mourir et qui entament leur parcours dans le Pō. En ce sens, les tuритириа ont un sens plus large que celui que nous avons développé jusqu'alors et représentent de cette façon un des aspects du continuum entre monde visible et invisible dans les conceptions cosmogoniques paumotu. Ces créatures étaient, par ailleurs, perçues comme malfaisantes. Or, aujourd'hui, si les tuриtupua sont toujours vus comme des entités émanant du $P o ̄$, ils ont désormais une connotation extrêmement positive liée à leur rôle de protecteur. Ce basculement de conception peut interpeller. Une explication se trouve peut-être dans le fait que les tuputupua se caractérisaient autrefois par une certaine dualité, Torrente écrit à ce sujet qu'ils ont

" pour caractéristique de posséder une puissance individuelle mana dont les manifestations peuvent être soit bénéfiques pour les vivants, soit dangereuses. " (Torrente, 2012: 225)

En définitive, le terme de tuputupua semble donc s'être simplifié avec le temps, pour ne plus désigner aujourd'hui que la réincarnation d'un ancêtre sous une forme animale.

En nous intéressant aux croyances semblables des autres sociétés du Pacifique, une divergence fondamentale apparaît dans cette affinité avec certains animaux. En effet, ces derniers sont plus souvent perçus non pas comme possédés par un ancêtre mais par une divinité de rang supérieur. La nature des tиритириа diffère par exemple des ata décrits par Raymond Firth à Tikopia. L'auteur écrit que, s'il existe bien

" une association particulière entre certains animaux et plantes et les intérêts religieux des habitants. Cette association est due au fait que les animaux et plantes sont utilisés comme des formes d'incarnations visibles par les divinités. » (Firth, 1930 : 291)

Ils sont donc le « reflet ", qui est une traduction possible du terme ata, d'une divinité à un instant précis. Les habitants de Tikopia interprètent une attitude anormale d'un animal comme le signe qu'il est alors possédé par une entité divine. Firth semble réfuter un lien avec les ancêtres, pourtant dans les textes qu'il retranscrit, nous pouvons constater que les habitants de Tikopia s'adressent aux ata en les appelant " $P u$ » ce qui signifie ancêtre dans la langue locale.

Si la nature des ata diverge de celle des tuputupua, nous ne savons que peu de choses sur leur rôle, sinon qu'ils sont souvent mal perçus par la population, les ata sous forme de chauve-souris détruisant les récoltes de fruits, ceux sous forme de baleines ou de crabes transportant des maladies, etc. En définitive, l'une des seules ressemblances avec les tuputupua concerne la manière dont la divinité prend possession du corps animal, puisque Firth explique que la divinité ne se change pas en animal mais entre dans son corps (Firth, $1930:$ 302).

Les ata sont à rapprocher des croyances décrites aux îles Tonga. Dans cet archipel,

"l'oiseau des tropiques, le martin-pêcheur, la mouette, la murène, le requin, la baleine et beaucoup d'autres animaux étaient sacrés parce qu'ils incarnaient l'esprit des dieux " (S.S. Farmer, in BatailleBenguigui, 1994 : 30)

Bataille-Benguigui note que : 
«les créatures zoomorphes dans lesquelles s'incarnaient les dieux étaient appelées $v a k a^{26}$, bateau ou réceptacle des dieux et les prêtres ou prêtresses étaient les taula otua (ancres des dieux). " (Bataille-Benguigui, $1994: 32)$

Cette dénomination taula otua correspond au terme taura que nous avons déjà évoqué. Ces vaka, comme les tuputupua, sont attachés à un groupe de parenté ou à un lieu géographique. Une autre croyance locale veut que :

" requin, poisson-chèvre et hareng viennent de Pulotu, le Paradis tongien [...]. Ces trois espèces étant des dons de dieux. [...] Dans les cas cités, le chef traditionnel local se considère comme le détenteur d'une espèce, si cette dernière passe dans ce qu'il croit être son territoire maritime. " (Bataille-Benguigui, 1988 : 189)

Cette croyance induit un autre type de relation entre homme et animal. En Nouvelle-Zélande, la relation entre animaux et entités spirituelles est connue sous le terme de aria. Elle se rapproche de celle observée à Tikopia ou à Tonga. Elsdon Best écrit à ce sujet que

" l'animal ou autre objet qui est la forme visible d'un atua est connue comme étant son aria. Ces aria, ou ces formes d'incarnations sont généralement des entités matérielles, telles que des animaux. " (Best, 1924 : 137)

La distinction entre ce qui relève du divin et ce qui relève de l'ancestral est parfois confuse chez certains auteurs. Ainsi, même si Best évoque dans sa définition des aria, les atua, c'est-à-dire des dieux n'ayant jamais eu forme humaine, il cite pour illustrer son propos l'ancêtre déifié Tamarau de la tribu Tuhoe qui était représenté sous la forme d'un lézard (Best, 1924: 137). Comme partout dans la zone, Best note que ces aria entretiennent des liens privilégiés avec certains clans ou individus.

À Hawaì i, nous trouvons beaucoup plus de proximité avec les aumakua. Herb Kane parle « d'esprits ancestraux " capables de prendre d'innombrables formes. Ils apparaissent aux vivants pour

" avertir d'un danger qui les menace, les réconforter dans des moments de stress ou de tristesse, ou leur être utile d'une autre manière. » (Kane, 2014 : 1)

Les différents récits recueillis par cet auteur, ainsi que par Martha Beckwith, sont similaires en tout point à ceux que nous avons recueilli aux Tuamotu. Au sujet de la forme que peut prendre un aumakua, Beckwith ajoute que comme Matirohe ou Te-aku-roa, "dans la plupart des cas, le aumakua a la capacité de prendre une forme soit humaine soit animale." (Beckwith, 1917 : 508)

Nous avons vu en citant plusieurs traditions recueillies par Jean Guiart qu'il existe en Mélanésie des croyances similaires notamment aux îles Salomon ou encore en Nouvelle-Calédonie où le terme qui leur est attribué est celui de rhë. Il serait trop long de commenter ici chacune de ces formes.

Revenons enfin en Polynésie française et plus particulièrement dans les îles de la Société, pour nous rendre compte que tãura semble bien être la traduction littérale du terme tuритириа. La définition d'un tāura faite dans l'ouvrage Porapora $i$ te fanau tahi entretient le doute quant à savoir s'il est l'incarnation de " l'esprit des dieux ou des ancêtres défunts" mais son rôle est semblable aux tuритириа, ils "veillaient sur les hommes mais aussi sur leur territoire ". Ils étaient également des tapao (signe)

«leur présence annonçait de bons ou de mauvais présages, l'arrivée d'une personne, la mort d'un proche, la guerre... » (Rattinassamy, 2001 : 51)

Les récits recueillis s'apparentent à ceux des tuputupua, comme celui de Tamaui dont

«l'esprit s'incarna dans un requin. " (Rattinassamy, $2001: 54)$

En Océanie, les relations homme - animal observées correspondent plutôt à la quatrième catégorie de la classification exposée dans notre tableau en amont. Les traditions de Turia, Matirohe, Te-aku-roa et du kaveu de Raroia le confirment. La seule variable concerne le fait de savoir si cette relation est établie avec un animal particulier ou une espèce toute entière. Il semble que les deux soient possibles comme l'écrit Beckwith à Hawaìi où

"dans certains cas, des individus sont l'objet d'un culte, dans d'autres tous les membres d'une espèce. » (Beckwith, 1917 : 506-507)

L'extrait du puta tupuna de Makemo (supra, p. 308) ainsi que les traditions relatives aux baleines sur cet atoll semblent également admettre cette possibilité.

\section{Culte et tapu}

Les récits dont nous avons traité livrent quelques détails qui peuvent amener à penser que la communication entre les êtres humains et

26. Nous avons vu plus haut que le terme vaka était connu à Napuka, où ce nom est donné aux «tuputupua qui ont un rôle de messagers " (Conte, $1988: 163)$. 
les tuритириа était réservée à certaines personnes pourvues de mana, les taura, ce qui rapprocherait cette pratique de celle d'un culte. Ainsi à Napuka, l'ancien Kape, qui avait comme tuputupua un poulpe,

« passait pour être détenteur d'un certain pouvoir surnaturel. » (Conte, 1988 : 163)

Il en va de même pour Kapea à Makemo, qui avait la capacité d'appeler et de guider les baleines. Cette communication est ritualisée, elle passe par une invocation dont la forme n'a malheureusement pas pu être définie. Parallèlement à ces invocations, certains objets pouvaient être utilisés pour entrer en contact avec le tuputupua. L'anecdote d'Anne Hervé à Hikueru a mis en lumière l'importance du monö̈. À Makemo, pour entrer en contact avec les baleines, un des tuputupua lié au gati Mahia, la personne chargée de monter sur le dos de l'animal s'enduisait de monö̈ rega ${ }^{27}$. Les reliques humaines avaient aussi un rôle dans cette communication, c'est pourquoi elles étaient précieusement conservées par les familles des personnes décédées.

Nous avons vu également que certains tuputupua étaient associés à un lieu rituel particulier, notamment Matirohe et le marae Patita. Toutefois, ce culte, s'il existe, ne semble pas indépendant de celui qui est rendu aux ancêtres dans l'archipel. Caillot parle d'une croyance

«singulière [...] qui enseignait que les esprits des morts ne pouvaient être heureux que s'ils étaient honorés et entourés de l'affection de leurs parents. " (Caillot, 1932 : 77)

Les rites sont accomplis par les membres de la famille et consistent en diverses offrandes et prières afin d'entretenir la communication avec le défunt, dans ce même esprit de continuité entre monde visible et invisible déjà évoqué.

Ce lien entre tuputupua et culte des ancêtres est confirmé à Hawai'i. Nous avons constaté que les aumakua sont une entité proche de celle des Tuamotu, différents auteurs décrivent les rites qui leur sont associés. Serge Dunis mentionne

"l'offrande de patates douces faite lors du premier empoissonnement » (Dunis, 1990 : 109)

qui leur était réservée. Ces offrandes sont confirmées par Beckwith :

" afin que le aumakua soit assez fort pour jouer son rôle de gardien, il doit recevoir des offrandes de prières et de sacrifices sous la forme de nourriture et boisson, appelées "feeding the spirit". " (Beckwith, 1917 : 505)
Elle ajoute dans sa description du culte rendu aux aumakua que :

« [ils] sont invoqués par des prières spéciales et ont des temples érigés pour leur culte. » (Beckwith, 1917 : 508)

Aux îles Salomon, on parle d'un requin nourri :

« par un homme particulier qui a établi une relation particulière avec la bête, en prenant la suite de ses pères et grand-pères. » (Guiart, in Clua et Guiart, 2015 : 40)

De la même façon voit-on Kape de Napuka nourrir son tuputupua sous forme de poulpe (Conte, $1988: 163$ ).

Le lien établi entre une espèce animale et une entité spirituelle (divine ou ancestrale) peut nécessiter l'instauration d'un tapu sur la pêche ou la chasse de l'espèce en question. Aux Tuamotu, il est difficile de savoir si ce tapu a existé. Les tuputupua correspondent généralement à un individu particulier, ce dernier était naturellement protégé mais son existence entraînait-elle nécessairement la mise en place d'un tapu sur l'espèce auquel il appartient ? Caillot écrit que les Paumotu :

"s'abstenaient de le tuer et de le manger " (Caillot, $1932: 85)$

mais une nouvelle fois son propos est imprécis puisque l'on ignore s'il fait référence à un individu ou à une espèce. Emory, en revanche, apporte une information importante :

«certaines familles ne consommaient pas de chair de paraoa (baleine) car ces dernières étaient des descendants de Ruatamahine, dont la mère avait adopté les deux baleines, Tutu-nui et Toga-mau-tutu, célèbres dans la légende de Kae " (Emory, 1975 : 215).

À Makemo, les récits traitant d'une affinité avec les baleines indiquent bien une capacité de communication avec les cétacés mais la finalité de cette relation est la mise à mort de l'un d'eux, dans une forme de réactualisation de la scène mythique entre Kae et la baleine Tutunui (voir à ce sujet Von Den Steinen, 2005 : 148).

Le tuритириа, lorsqu'il est un individu isolé, est évidemment respecté et sa mise à mort provoque la colère du groupe social auquel il est affilié, nous l'avons vu à Takume avec Te-aku-roa. Dans les traditions de Hao, au sud de la zone Tapuhoe, il est fait état de la rencontre entre le grand ariki Munanui et des étrangers venus de l'est (Caillot, 2008 : 38 ; Audran, 1918 : 92-93). Munanui sert comme repas à ces étrangers du chien ce qui entraîne leur fureur car «cet animal était le roi vénéré de leur clan " (Audran). On peut légitimement penser que le terme tuритириа devrait 
être substitué à celui de roi. Le chien est en effet connu comme tel dans l'archipel. Ottino mentionne notamment le chien Hauatua à Rangiroa (Ottino, 1965 : 111).

Ailleurs dans le Pacifique, il est évident que lorsque l'entité est assimilée à un ancêtre, le tapu est de mise comme l'a conclu Handy pour Tonga ou les îles Cook (Handy, 1968 : 55). Dans les autre cas, ceux où l'entité est perçue comme l'incarnation visible d'une divinité, Firth note :

« interférer avec la créature, la tuer ou la manger, est perçu comme un acte hostile, non pas envers le groupe ou l'un de ses membres, mais envers la divinité qui possède la créature à cet instant, et qui punira le sacrilège. » (Firth, 1931 : 387)

\section{Conclusion}

Nous avions suggéré, dans notre introduction, que la croyance aux tuритириа pouvait s'apparenter à une forme de totémisme. Au final, malgré de nombreuses similitudes, elle ne s'inscrit pas tout à fait dans ce cadre, en tout cas si l'on en juge par certaines définitions classiques, comme celle de Rivers, c'est-à-dire une croyance caractérisée par :

"la coalescence de trois éléments. Un élément social : connexion d'une espèce animale ou végétale, ou d'un objet inanimé avec un groupe défini de la communauté et typiquement avec un groupe exogamique ou clan. Un élément psychologique : croyance en une relation de parenté entre les membres du groupe et l'animal s'exprimant souvent par l'idée que le groupe humain en est issu par filiation. Un élément rituel : respect témoigné se manifestant typiquement dans l'interdiction de manger l'animal. » (Lévi-Strauss, 1962:15-16)

La croyance aux tuputupua repose, en effet, principalement sur une affinité avec un individu animal et non une espèce. Cette divergence fait que ce type de relation a souvent été considéré comme n'appartenant pas au totémisme (cf. supra, tab. 1), une position vivement critiquée par Lévi-Strauss qui notait que :

« on ne viendra pas à bout du prétendu problème totémique en imaginant une solution à champ d'application limité, puis en manipulant les cas rebelles jusqu’à ce que les faits veuillent bien s'incliner, mais en atteignant d'emblée un niveau suffisamment général pour que tous les cas observés puissent y figurer comme des modes particuliers. » (Lévi-Strauss, 1962 : 115)

Le débat est sans doute plus large que la question de l'appartenance ou non au totémisme car cette croyance que nous avons étudiée nous interroge plus généralement sur le rapport entre homme et nature au sein de la société paumotu et sur l'existence d'une forme de circulation de l'un à l'autre. Ainsi Philippe Descola écrivait :

« [qu']en postulant une distribution universelle des humains et des non humains dans deux domaines ontologiques séparés, nous sommes d'abord bien mal armés pour analyser tous ces systèmes d'objectivation du monde où une distinction formelle entre la nature et la culture est absente. La nature n'existe pas comme une sphère de réalités autonomes pour tous les peuples, et ce doit être la tâche de l'anthropologie que de comprendre pourquoi et comment tant de gens rangent dans l'humanité bien des êtres que nous appelons naturels. » (Descola, $2002: 14$ )

C'est bien dans cette direction que semblent nous amener nos traditions relatives aux tuputupua, dans lesquelles l'entité en question illustre une continuité entre homme et nature, entre monde visible et invisible.

Aux Tuamotu, les tиритириа constituent la base d'une croyance encore bien connue de nos jours, contrairement aux dires de Caillot affirmant qu'elle n'existait déjà plus lors des premiers contacts avec les Européens. Kane dit la même chose à Hawai'i où :

"l'acceptation du Christianisme n'a pas forcé l'abandon des croyances relatives aux aumakua." (Kane, $2014: 2$ )

En cela, ils forment une source d'étude intéressante pour l'anthropologie aux Tuamotu car, tandis que ce qui a trait aux marae ou aux dieux anciens a été en grande partie effacé par plus de 150 ans de présence chrétienne, des informations relatives à ce sujet ont su être conservées jusqu’à aujourd'hui. Plus que de simples souvenirs, nous avons relevé plusieurs anecdotes récentes qui ont démontré l'aspect contemporain de cette croyance, puisque des Paumotu aujourd'hui encore invoquent leur tuритириа quand ils sont en danger, notamment en mer. Cette situation, au sein de sociétés fortement imprégnées de christianisme, est un des aspects illustrant la cohabitation au quotidien de croyances traditionnelles et chrétiennes dans l'actuelle Polynésie française.

\section{BIBLIOGRAPHIE}

Audran Hervé, 1918. Les Hivas et les Tavas. Traditions of and notes on the Paumotu (Tuamotu) Islands, Part II, Journal of the Polynesian Society 27, pp. 90-92.

Babadzan Alain, 1982. Naissance d'une tradition. Changement culturel et syncrétisme religieux aux îles Australes, Paris, Travaux et documents de l'orstom. 
Bacchet Philippe, Thierry Zysman et Yves LeFÈvre, 2006. Guide des poissons de Tahiti et ses illes, Pirae (Tahiti), Au vent des Îles.

Bataille-Benguigui Marie-Claire, 1988. The fish of Tonga: prey or social partners?, Journal of the Polynesian Society 97, pp. 185-198.

—, 1994. Le Côté de la mer. Quotidien et imaginaire aux îles Tonga, Bordeaux-Talence, Presses universitaires de Bordeaux.

Bескшітн Martha Warren, 1917. Hawai ian shark aumakua, American Anthropologist 19, pp. 503-517.

BEsT Elsdon, 1924. Maori religion and mythology: being an account of the cosmogony, anthropogeny, religious beliefs and rites, magic and folk lore of the Maori folk of New Zealand. Section I, Wellington (NZ), Skinner, Government printer.

CaIllot Eugène, 1932. Histoire des religions de l'archipel Paumotu avec des tableaux de la société indigène et des traditions anciennes recueillies, publiées et traduites en français et commentées, Paris, Ernest Leroux.

—, 2008. Mythes, légendes et traditions des Polynésiens, Pappeete, Haere Po.

Charpentier Jean-Michel et Alexandre FranÇOIs, 2015. Atlas linguistique de la Polynésie française, Université de la Polynésie française, De Gruyter Mouton.

Clua Éric et Jean Guiart, 2015. Requins d'Océanie : ethno-écologie d'un prédateur marin, [Papeete], Te Pito o te Fenua.

Conte Éric, 1988. L'exploitation traditionnelle des ressources marines à Napuka (Tuamotu, Polynésie Française), thèse de doctorat, Université Paris 1 , multigr. (2 vol.).

-, 1990. Archéologie des Tuamotu (Polynésie française). Prospection de dix atolls du centre de l'archipel. Inventaire archéologique de la Polynésie française, Punaauia (Tahiti), Département archéologie du CPSH.

Craig Robert, 1989. Dictionary of Polynesian mythology, New York, Greenwood Publishing Group.

Descola Philippe, 2002. L'anthropologie de la nature, Annales. Histoire, sciences sociales 1, pp. 9-25.

Dunis Serge, 1990. Ethnologie d'Hawaì. Homme de la petite eau, femme de la grande eau, Paris, Presses Universitaires Créoles/L'Harmattan.

Elbert Samuel H. et Mary Kawena Pukui, 1986. Hawaiian dictionary: Hawaiian-English, English-Hawaiian, Revised and enlarged edition, Honolulu, University of Hawai'i Press.

Emory Kenneth, 1940. Tuamotuan concepts of creation, Journal of the Polynesian Society 49, pp. 69-136.
-, 1975. Material culture of the Tuamotu archipelago, Honolulu, Dept. of Anthropology, Bernice Pauahi Bishop Museum.

FIRTH Raymond, 1930. Totemism in Polynesia, Oceania 1 (3), pp. 291-321.

—, 1931. Totemism in Polynesia (Continued), Oceania 1 (4), pp. 377-398.

Guiart Jean, 2008. Myth in the islands, in S. Dunis, Sexual Snakes, Winged Maidens and Sky Gods, Papeete, Haere Po, pp. 11-49.

—, 2012. Religions d'Océanie, Papeete, Imprimerie Fastprint.

Gunson F. Allan, 1982. Female pollution in Polynesia?, Journal of the Polynesian Society 91, pp. 335-381.

Handy E.S. Craighill, 1968. Traces of totemism in Polynesia: theories of embodiment of tutelary spirits in animate and inanimate forms, Journal of the Polynesian Society 77, pp. 43-56.

Henry Teuira, 1988. Tahiti aux temps anciens, Paris, Musée de l'Homme, Publications de la Société des Océanistes n 1 (http://books.openedition.org/sdo/1402?lang=fr).

Hervé Anne, 1948. Magie et sorcellerie aux Tuamotu chez les indigènes de l'archipel Paumotu, Journal de la Société des Océanistes 4, pp. 49-56 (http://www.persee.fr/docl jso_0300-953x_1948_num_4_4_1591).

Kane Herb Kawainui, 2014. The 'Aumakua: Hawai'ian ancestral spirits (consulté sur http://dlnr.Hawai'i.gov/sharks/files/2014/07/ APaperbyHerbKane.pdf).

LÉvi-STrauss Claude, 1962. Le totémisme aujourd'hui, Paris, Presses universitaires de France.

Otтino Paul, 1965. Ethnohistoire de Rangiroa (archipel des Tuamotu) [Publication provisoire], ORSTOM.

Rattinassamy Martine, 2001. Porapora i te fanau tahi: mille ans de mémoire, Papeete, Ministère de la Culture de Polynésie française, Cahiers du Patrimoine $n^{\circ} 2$.

Stimson Frank, 1933. Tuamotuan Religion, Bernice Pauahi Bishop Museum, Honolulu, Bulletin 103.

Stimson Frank et Donald Stanley Marshall, 2008. A dictionary of some Puamotuan dialects of the Polynesian language, Papeete, Société des études océaniennes.

Torrente Frédéric, 2012. Buveurs de mers, mangeurs de terres. Histoire des guerriers d'Anaa, atoll des Tuamotu, Papeete, Haere Po. 